\title{
APPLICATIONS OF ATOMIC FORCE MICROSCOPY IN THE PHARMACEUTICAL SCIENCES
}

\section{J.T. Thornton}

Digital Instruments, Veeco Metrology Group, 223 Wilmington-West Chester Pike, Suite 114, Chadds Ford, PA 19317

Surface interactions play a key role in the design and delivery of a pharmaceutical product. After the steps of drug formulation, it is important for the design and evaluation of the fabrication process to perform further tests, like microscopic evaluation. Since Atomic Force Microscopy (AFM) provides the ability to investigate surface structure at nanometer-to-subangstrom resolution in ambient and liquid environments, it has been applied to a wide range of studies in pharmaceutical research.

For many years, the AFM has been used routinely in many scientific fields, commonly investigating DNA, proteins, and cells in biological studies; structure and component distribution in polymer science; $\mathrm{pN}$ force interactions and surfactant behavior in colloid science; and physical/mechanical properties and fabrication variables in the material sciences. Pharmaceutical research often consists of a combination of these scientific branches, which makes it a viable field for the application of AFM. Plus, the ability of AFM to provide high resolution, three-dimensional surface structure regardless of sample conductivity makes it a powerful complement to other common analytical techniques currently available ${ }^{1}$

The applications of AFM to pharmaceutical research range from drug discovery to tablet manufacturing. In drug research, AFM has been applied to investigating gene delivery vehicles ${ }^{2}$ (figure 1), drug interactions ${ }^{3,4}$, disease mechanisms ${ }^{5}$, and crystal growth ${ }^{6}$. Many of these studies are conducted in solution environments that mimic physiological conditions. Once the drugis produced in crystalline and amorphous forms, it is typically processed into particles by micronization, spray drying, or supercritical fluid techniques. The drug particle size and morphology can be characterized by AFM, as well as the granules and inhalation particles produced by mixing the drug with other components $^{7}$. Force studies between particles and gelatin capsule surfaces have been conducted to study their interaction mechanisms which can affect the distribution of particles from dry particle inhalers $^{8}$. Characterization of coatings used throughout tablet production are conducted to tailor the fabrication parameters to achieve the desired morphology (figure 2). Dissolution mechanisms have also been visualized in situ to study rate and behavior in different fluid environments ${ }^{9}$. Thus, AFM has been used throughout the drug fabrication process to characterize morphology and interaction mechanisms.

References:

[1] Russell, P., et al., Microscopy and Analysis 49 (2000) 5.

[2] Martin, A.L., et al., FEBS Letters 480 (2000) 106

[3] Coury, J.E., et al., Proc. Nat. Acad. Sci. USA 93 (1996) 12283.

[4] Rotsch, C., Radmacher, M., Biophys. J. 78 (2000) 520.

[5] Yip, C.M., McLaurin, J., Biophys. J. 80 (2001) 1359.

[6] Yip, C.M., Ward, M.D., Biophys. J. 71 (1996) 1071.

[7] Li, T., Park, K., Pharm. Res. 15 (1998) 1222. 
[8] Willing, G.A., et al., J. Colloid Interface Sci. 226 (2000) 185.

[9] Danesh, A., et al., Pharm. Res. 18 (2001) 299.

[10] Images courtesy Sophia Hohlbauch Digital Instruments, Veeco Metrology Group; Samples courtesy of V. Trubetskoy, Mirus Corporation.

[11] Image courtesy Debra Cook, Digital Instruments, Veeco Metrology Group; Sample courtesy ALZA Corporation.

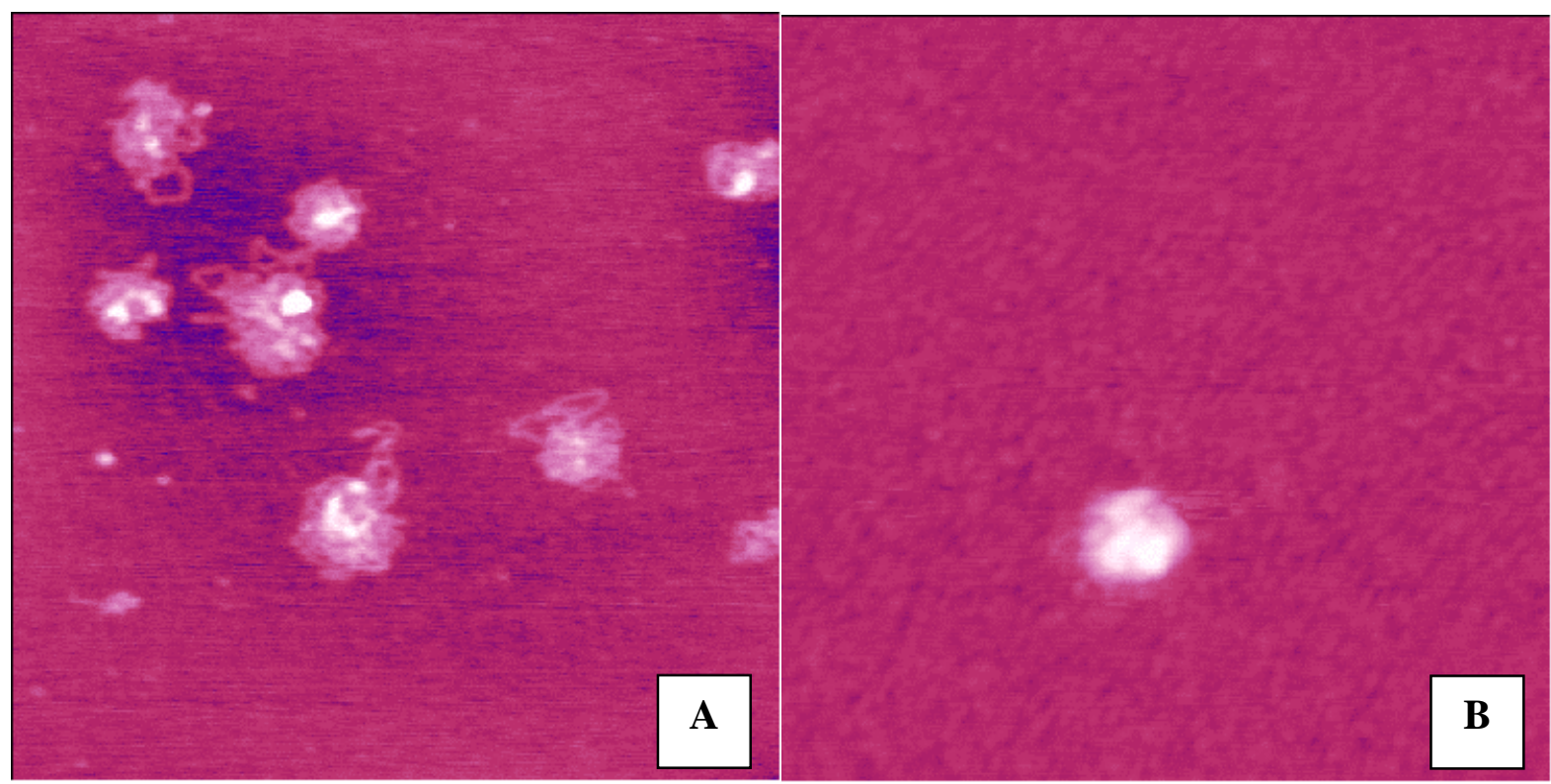

Figure 1. Two different condensed states of DNA from a study of non-viral gene delivery vehicles: a) Condensed negatively charged with $0.2 \mathrm{mM} \mathrm{NiCl}$, b) Condensed positively charged. Depending on the formation mechanism, the condensates are tightly packed or slightly unraveled ${ }^{10} 1 \mu \mathrm{m}$ scans.
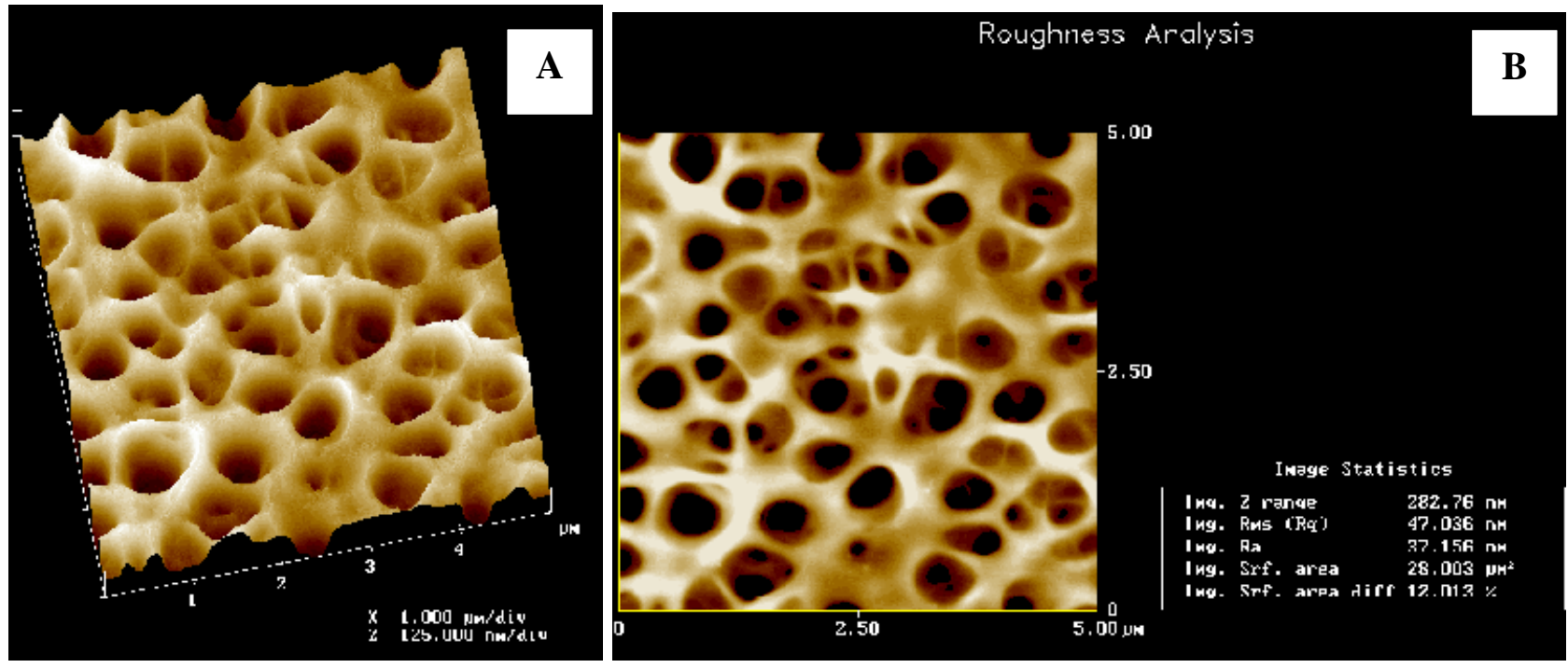

Figure 2. Tablet coating which functions as a membrane for controlled-release applications showing complex pore structure. Surface structure, roughness and surface area are characterized in (b) ${ }^{11}$. $5 \mu \mathrm{m}$ scan. 